\title{
El temperamento sí importa: Características psicológicas necesarias para liderar escuelas
}

\author{
Temperament Matters: Psychological characteristics needed to lead \\ schools
}

\section{Roberto Barrientos Mollo ${ }^{1}$}

\section{Revista \\ Educación y Sociedad}

Citar como: Barrientos, R. (2021). El temperamento sí importa: Características psicológicas necesarias para liderar escuelas. Revista Educación y Sociedad, 02(03), 48-57. doi: 10.53940/reys.v2i3.60

Artículo recibido: 10-02-2021 Artículo aprobado: 20-06-2021 Arbitrado por pares

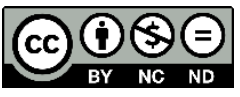

\section{ACEES}

\section{Resumen}

El liderazgo educativo es un elemento clave para la transformación y mejora de la educación. En este artículo se analizan las características psicológicas necesarias que deben tener los líderes educativos para realizar cambios en educación. Las características psicológicas que los líderes deben desarrollar a un nivel más allá de lo común son la autoeficacia, la resiliencia y el optimismo. Se explica el significado de cada una y se ofrecen recomendaciones para su desarrollo tomando como referencia la literatura y la experiencia de directores de escuelas. Se recomienda a los reclutadores de directores tener en cuenta estas características en el proceso de selección de los mismos.

Palabras clave: liderazgo educativo, mejora escolar, cambio educativo, autoeficacia, resiliencia, optimismo

\section{Abstract}

Educational leadership is a key element for the transformation and improvement of education. This article analyzes the necessary psychological characteristics that educational leaders must have to make changes in education. The psychological characteristics that leaders must develop beyond the ordinary are self-efficacy, resilience, and optimism. The meaning of each one is explained and recommendations for their development are offered, taking as a reference the literature and the experience of principals. Principals recruiters are encouraged to consider these characteristics in the director selection process.

Key words: educational leadership, school improvement, educational change, selfefficacy, resilience, optimism 


\section{Introducción}

Liderar una escuela o un sistema educativo en tiempos inciertos y desafiantes no es fácil. Para ello se necesitan determinados conocimientos, habilidades y características personales. En el Liderazgo sí importa: aportes para una mejor comprensión de la dirección escolar (Barrientos, 2015) realicé una síntesis de las competencias, recursos sociales y psicológicos que debe tener un director o directivo de escuela basado en la evidencia existente en ese momento. En el presente documento desarrollo las características psicológicas mencionadas en ese artículo, que son la autoeficacia, resiliencia y optimismo. En cada característica se ofrece una explicación sobre su naturaleza y recomendaciones para su desarrollo recogiendo aportes de la investigación y experiencia de líderes educativos.

Los líderes educativos deben de poseer estos recursos psicológicos a un nivel más allá de lo común (Fullan, 2013). Por ello es importante realizar este análisis puesto que, en gran parte de los procesos de selección, desarrollo y evaluación de desempeño de líderes educativos no se los toma en cuenta. De esta manera se comete un error con consecuencias graves para la vida de miles de instituciones educativas y millones de estudiantes, puesto que se tiene menores posibilidades de tener resultados de aprendizaje. De allí que los procesos de reclutamiento de directivos deben de dar un lugar especial a la evaluación de estas características. En segundo lugar, si ya se tiene directivos contratados que no la tienen a un alto nivel, lo que queda es aprenderlas, dado que "estas habilidades [resiliencia, optimismo y eficacia] se pueden aprender, pero no tan rápidamente y no es tan fácil como otras habilidades" (Leithwood, 2012). Por lo que deben de ser parte de las temáticas a trabajar en los espacios de entrenamiento y desarrollo profesional de directivos.

La autoeficacia, resiliencia y optimismo están conectadas entre sí de tal manera que se puede decir que son caras de una misma moneda, de esta manera el desarrollo de una característica retroalimenta a la otra. La característica psicológica que tiene más literatura desarrollada es la autoeficacia. Al respecto son icónicos los estudios de Bandura (1977, 1995; 1993). Según Leithwood (2012), un indicador para corroborar si una persona tiene desarrollada una o las tres es la actitud de tomar iniciativas. Por lo que se puede afirmar que la persona que sale al encuentro de las necesidades institucionales, aunque no esté escrito en su función, probablemente, tiene desarrollada alguna de ellas.

\section{La autoeficacia}

La autoeficacia es la creencia de una persona en su capacidad de tener éxito en una situación o tarea particular ( Leithwood, Louis, Anderson y Wahlstrom, 2004). Es una creencia sobre la propia capacidad, no la capacidad real. Sin embargo, como las creencias tienen un poder en nuestras acciones, ocurre como la profecía autocumplida, si se cree firmemente que se puede hacer algo, hay muchas probabilidades de que lo haga realmente. Por eso es una habilidad importante en los líderes educativos que tienen que enfrentar en el día a día muchos imprevistos y desafíos.

Para Bandura (1977) es la creencia de un individuo en su capacidad para ejecutar comportamientos necesarios para producir logros de desempeño específicos. De esta manera, las personas estarán más motivadas si perciben que sus acciones pueden ser eficaces. "Las expectativas de eficacia determinan cuánto esfuerzo dedicarán las personas y cuánto tiempo persistirán frente a los obstáculos y las experiencias adversas. Cuanto más fuerte sea la autoeficacia percibida, más activos serán los esfuerzos" (p.193). De esta manera se genera una suerte de círculo virtuoso, si creo que soy bueno en algo, entonces lo haré con más confianza, esta confianza me motivará a seguir haciéndolo y me dará las fuerzas para perseverar ante las dificultades. Es por ello que la autoeficacia influye a nivel cognitivo, afectivo y volitivo. Es muy probable que una persona con un alto nivel de autoeficacia para una determinada tarea sea también optimista y resiliente en ese ámbito. 
La eficacia es más que sólo ser positivo u optimista frente a un desafío, se trata de ser consciente de que se tiene control de esa tarea o encargo. Es pensar "Tengo control y, por eso, lo puedo hacer bien".

Esa creencia sobre sí mismo otorga a la persona seguridad. La seguridad es una cualidad clave en los líderes. Los equipos necesitan seguridad y claridad de parte de sus encargados, a pesar de que éstos últimos no lo tengan claro en un momento dado. Si no se ofrece seguridad a los equipos se generará incertidumbre en los mismos y la incertidumbre genera parálisis. Y lo que menos se necesita en ambientes dinámicos y cambiantes como son las escuelas y sistemas educativos es paralizarse.

\section{Recomendaciones para su desarrollo}

\section{La práctica abundante}

Como dice Leithwood y McCullough "Las experiencias de dominio, o los éxitos y fracasos pasados individuales con una tarea, tendrán fuertes efectos en los sentimientos sobre el logro de tareas futuras similares" (2020, p. 67). Por lo que se desarrolla con mucha práctica como toda habilidad y observando los efectos de las propias acciones. Existen muchas dimensiones de la gestión de un líder educativo: la gestión de los equipos docentes, el clima de escuela, la gestión y trato con padres de familia, la gestión de los conflictos en la escuela o UGEL, la administración escolar, etc. Nadie que asume una escuela es bueno en todas esas dimensiones. Se sugiere identificar aquella en la que se es bueno y enfocarse para desarrollar esa confianza. Luego elegir una habilidad en la que no se es tan bueno y proponerse metas cortas y pequeñas. De esta manera ocurrirá un dominio progresivo y se irá fortaleciendo la autoeficacia. Repetir el éxito en determinadas tareas aumenta los niveles de autoeficacia mientras que los fracasos repetidos la disminuyen.

La directora del colegio San Marcos, Nancy Rivera, comparte su experiencia en la implementación de un nuevo proyecto de aprendizaje:

Este año me propuse implementar un proyecto de aprendizaje a nivel institucional. En la primera reunión cuando lo compartí tratando de contagiar las ventajas para nuestros estudiantes que tendrían la oportunidad de vivenciar un aprendizaje profundo. No tuve mucho eco. Hubo mucha resistencia de implementar algo que no se había hecho antes desde la planificación curricular. Esto no me desanimó, al contrario, empecé a preparar materiales, talleres internos y participación externa en talleres para que los docentes conozcan más al respecto. Se hizo toda una campaña para empoderarlos respecto a ese proyecto de aprendizaje. Para ello en cada encuentro que tenía con mis docentes daba algo que había preparado con mucho esfuerzo (taller, materiales y lecturas). A cambio, iba logrando compromisos a partir de lo dialogado o trabajado. Así que después de casi cuatro meses se logró implementar el proyecto.

Muchos docentes sobre todo los que no estaban convencidos de implementarlo se mostraron satisfechos con el logro alcanzado. Han pasado ya cinco meses de ello y todavía siguen citando la experiencia. Lo que para mí es una evidencia de un aprendizaje profundo que han vivenciado los docentes. Y de que un directivo debe de saber perseverar cuando algo vale la pena (Comunicación personal, 20 de noviembre de 2020).

Nancy perseveró porque sabía que era algo bueno y porque tenía confianza en sí, sabía que en otras oportunidades había logrado movilizar al equipo docente. Esa frase interna de "Ya lo hice antes, creo que puedo hacerlo bien ahora" es la autoeficacia. Y eso se logra con mucha práctica. En este ejemplo se pueden ver las tres características en acción: la confianza o eficacia de Nancy, su optimismo, saber que al final las cosas saldrían bien y resiliencia, cuando dice "pero eso no me desanimó". 


\section{Actuar con seguridad}

El líder debe transmitir seguridad. Se recomienda transmitir esta seguridad, a pesar que no la sienta en un primer momento (Sutton, 2011). Andy Grove logró hacer el salto para que Intel pase de producir memorias a microprocesadores de computadoras, posicionándolo en este rubro como el mejor del mundo. Afirma que el gestor debe siempre de mostrar seguridad frente a las mil y un demandas y decisiones que tiene que tomar. Para explicar la situación del mismo usa como imagen al protagonista de la serie Los Soprano, Tony Soprano, quien a pesar de todas las tensiones e inseguridades internas supo mantener el rumbo de su organización y de su propio hogar.

Es por ello que al inicio el líder quizá deba actuar esa seguridad hasta que ésta se convierta en real. Para ello es necesario que frente a las consultas en las que hay que tomar decisiones día a día decir con claridad "sí,sí; no, no o no lo sé". Lo que menos necesitan los equipos es titubeo. Los equipos necesitan que sus responsables sean seguros, como un capitán que guía un barco. Su objetivo es que el barco llegue al puerto, para ello tiene que tener claro, que la gente está en su sitio y me mire como aquél como el que lo impulsó y lo animó.

\section{La modelización}

Otra manera de desarrollar autoeficacia es por modelización. Es decir, se debe tener referentes de los cuales aprender. La recomendación de juntarse con los mejores se aplica para esta situación. Debe juntarse con los mejores en la habilidad que se desea aprender, tomar lo mejor y ponerlo en práctica. Para ello la estrategia de la mentoría es de gran ayuda. La clave de una buena mentoría está en elevar la vara y ofrecer apoyo. El mentor debe tener altas expectativas y exigir:

El empujoncito de un mentor lleva a tirar la cuerda, lo cual crea un momento de autoconocimiento. Lo que puede resultar ilógico de esta visión de la mentoría es la parte sobre el empujoncito. Requiere que el mentor exponga a su protegido al riesgo. $Y$ esto puede ser antinatural; nuestro instinto con la gente que nos importa es protegerlos de cualquier riesgo. (...) La promesa de ir más allá no es el éxito, sino aprender. Es el autoconocimiento. Es la promesa de obtener las respuestas a algunas de las preguntas más importantes y desconcertantes de nuestras vidas: ¿Qué queremos? ¿Qué podemos hacer? ¿Quién podemos ser? ¿Qué podemos soportar? (Heath \& Heath, 2018, p.78).

Entonces, como líder del cambio se debe pedir y ofrecer mentoría. Esa persuación verbal que recibe la persona implica recibir aliento, exhortación, así como una retroalimentación continua de un colega creíble, de confianza y respetado de su jurisdicción.

Otro tipo de modelización es lo que Leithwood y McCullough (2020) llaman "experiencias vicarias". Las experiencias vicarias son aquellos aprendizajes que surgen de la visualización continua de un desempeño exitoso de otras personas. Uno aprende de los errores y éxitos de otros, como si fueran suyos.

La movilización emocional es otra estrategia para desarrollar la autoeficacia. Ésta puede ocurrir como "respuesta a un otro inspirador que ayuda a elevar los estándares y aspiraciones de los líderes y les ayuda a ver la relación entre las metas de su organización y propósitos sociales y morales más amplios" (Leithwood y McCullough, p. 67, 2020).

En síntesis, la creencia positiva sobre el propio desempeño en una situación dada se puede aprender y desarrollar. Para ello se debe: 1) Realizar mucha práctica deliberada enfocada en lo que se sabe hacer y en lo que no se sabe hace aún, 2) Si al inicio no se tiene esa seguridad, actuar y transmitir la seguridad y confianza que los equipos necesitan y 3 ) Juntarse con los mejores para ser mentoriado y ofrecer mentoría a otros. 


\section{La resiliencia}

La resiliencia es la conciencia de que las cosas no siempre saldrán como queremos, pero que podremos solucionarlas (Leithwood, 2012). Esta capacidad está muy relacionada con la persistencia, la misma que permite perseverar y mantenerse firme a pesar de que las cosas no vayan bien. El núcleo de la resiliencia consiste en saber recuperarse del fracaso y no sólo eso, y, mientras se recupera, ser capaz de superar las metas iniciales.

El trabajo del líder educativo es mover a la organización un paso más allá. Dicho movimiento es cambio y a nadie le gusta moverse de su zona de confort, siempre generará un tipo de dolor e incomodidad. Es por ello que los líderes deben estar listos para que no los miren con buena cara en las primeras semanas o meses de implementación (Fullan, 2013). Para ello es necesario un tipo de temperamento dispuesto a recibir miradas incómodas al inicio de los cambios. Dicho temperamento le permite mantenerse firmes hasta que las personas empiecen a ver los frutos de los nuevos hábitos o prácticas organizacionales para bien de los estudiantes. En el caso presentado de la directora Nancy su resiliencia le permitió perseverar en la implementación del proyecto de innovación a pesar de la resistencia inicial, con resultados positivos al final.

La resiliencia y la terquedad no son sinónimos. Por ello el líder educativo que inició un cambio de prácticas en el aula, escuela o gobierno local educativo necesita estar muy atento a la información que le brinda la realidad. Esta escucha atenta a la realidad le permite darse cuenta si es que no está funcionando la estrategia y es necesario detenerla o cambiar de rumbo (pivotar) o, tomar conciencia de que es solo una incomodidad inicial, por lo que es necesario perseverar. De allí la importancia de que la resiliencia vaya de la mano con altas capacidades y mecanismos de escucha a los usuarios.

Un ejemplo de firmeza y empatía profunda de los directores lo muestra César Reina, director del colegio María Parado de Bellido del Centro de Lima. La experiencia que a continuación comparte es sobre el nuevo hábito de usar las herramientas de la plataforma virtual de la Estrategia Aprendo en Casa (AeC). Los docentes preferían la versión de $\mathrm{AeC}$ que se presentaba en televisión y pocos usaban la plataforma web de la estrategia por ser más complicada. Al final fruto de la persistencia de los directivos se logró pasar al uso de los materiales de la plataforma web. A continuación el comentario del director César:

Cuando los docentes tenían que ingresar a la plataforma web de aprendo en casa, muy pocos lo hacían porque contenía mucho texto. Todos o casi todos iban por la versión de televisión, porque supuestamente era más fácil que los niños vean el programa. Luego el profesor se reunía con ellos y hablaba de lo que observaron. Esta estrategia aparentemente fácil, podía ser para uno o dos días, pero no para realizarlo de lunes a viernes y durante todos los meses. Es más complejo porque requiere mucha creatividad del docente, tener una amplia cultura y la versatilidad para planificar en el mismo momento una ficha de trabajo. Entonces, aquello que les costaba mucho en la manera presencial se convirtió de a pocos en la principal herramienta y estrategia [...] El docente miró las estrellas, se enfocó en aquello que les daría mejores resultados y fortalecería los espacios de interacción con sus pares. Así mismo, podrían entender el lenguaje de las normas (Comunicación personal, 10 de noviembre de 2020).

\section{Recomendaciones para su desarrollo}

Además de las recomendaciones sugeridas para el desarrollo de la autoeficacia, se sugiere, en primer lugar, enfocarse en lo que depende de uno y no de factores ajenos. En segundo lugar, tener pocos objetivos y enfocar la voluntad en ellos para que los resultados vayan apareciendo. Estos pocos objetivos tienen que estar enfocados en la buena pedagogía.

Nancy Rivera, directora del colegio San Marcos, comparte sus estrategias para desarrollar esa persistencia: 
Cuando inicia una semana tengo claro lo que voy a realizar y lograr en ese tiempo. Lo tengo en el planificador que se revisa diariamente, poniendo check de logrado. Esto me motiva de tal manera que, por muy abrumador que parezca lo que se viene en la semana, sí es posible lograrlo; y me acuesto con esa satisfacción de que lo pude hacer. Cuando algo sale mal lo comparto con alguien en quien confío. Es en ese diálogo o escucha de mí misma cuando recreo lo sucedido. De esta manera voy viendo algunas posibilidades para replantear lo que salió mal y mejorarlo o volver con fuerza si es que es necesario insistir en ello (Comunicación personal, 10 de noviembre de 2020).

Esta directora toma energía de los pequeños logros realizados durante el día. Además, el tener espacios de diálogo con otra persona de confianza le ayuda tener una nueva mirada de lo sucedido, para poder seguir intentándolo, a pesar de los fracasos iniciales. Entonces, una recomendación para fortalecer la resiliencia es apoyarse en otros para contarle el fracaso o dificultad que se tuvo en el día. De esta manera se empieza con fuerza el siguiente día, hasta que se visibilicen resultados.

\section{El optimismo}

El 12 de julio de 1988 Francia superó a Brasil en Copa del Mundo de fútbol. Ese día los hombres franceses disfrutaron de una tasa de mortalidad cardiovascular más baja que la media de los otros días entre el 7 y el 17 de julio, pero las mujeres francesas no. Los médicos no saben por qué disminuyeron los ataques cardíacos fatales; los investigadores opinan que el responsable podría ser un estallido de optimismo (Harvard Health Publishing, 2008).

El optimismo es aquella "disposición de que buenas cosas sucederán", generalmente, cuando se piensa así, esas cosas buenas suceden (Scheier et al., 1999). Para Leithwood y McCullough sería "la expectativa habitual de éxito en los esfuerzos de uno por abordar los desafíos y enfrentar el cambio ahora y en el futuro" (p. 56, 2020). Lo que se puede sintetizar en una expectativa habitual de éxito.

Esta disposición positiva ante los acontecimientos ha demostrado tener una relación con mayores logros en el mundo de la salud y en el mundo de la gestión.

\section{El optimismo en la salud}

Los estudios muestran que el optimismo es un factor protector y un factor preventivo. Con respecto al optimismo como factor protector ayuda a recuperarse de cirugías, así mismo tener los menores síntomas en el caso de infecciones virales. Respecto al optimismo como factor preventivo, ayuda a prevenir enfermedades como la presión arterial.

Un ejemplo del optimismo como factor protector es un estudio con 309 pacientes que serían sometidos a una operación de bypass de arteria coronaria. Estos pacientes fueron sometidos a un seguimiento de seis meses. Se descubrió que los pacientes optimistas tenían la mitad de probabilidades de requerir una nueva hospitalización respecto a los pesimistas (Scheier et al., 1999).

Otro ejemplo del optimismo como factor preventivo se puede ver en un estudio finlandés realizado en 616 hombres a los que se hizo seguimiento por 4 años. El estudio mostró que los pesimistas tenían el triple de posibilidades de desarrollar hipertensión arterial. Se controló otras variables como actividad física, consumo de cigarro, abuso de alcohol y obesidad. Es decir, el optimismo resultó un factor relevante para la salud de estas personas ( Everson, Kaplan, G, Goldberg y Salonen, 2000).

En otro estudio se introdujo un virus de la gripe a 196 jóvenes saludables y descubrieron que los jóvenes con visión optimista tenían menos probabilidades de desarrollar síntomas virales respecto de sus pares con visión pesimista ( Cohen, Doyle, Turner, Alper y Skoner, 2003). Las investigaciones muestran que una perspectiva optimista en una etapa temprana de la vida puede predecir una mejor salud y una menor tasa de muerte durante los períodos de seguimiento de 15 a 40 años. 
Por lo tanto, el optimismo es un factor protector y preventivo para una buena salud.

\section{El optimismo y la gestión}

El optimismo es una disposición clave para el desarrollo de una buena gestión (Davis-Blake y Pfeffer, 1989). Solo con una visión o disposición positiva sobre lo que puede ocurrir es que se puede gestionar entidades con estatus quo bastante enraizados, como son las escuelas y sistemas educativos. Es por ello que la actitud del líder es clave, porque tiene mayor posibilidad de contagiar hacia abajo su visión de las cosas ( Chemers, Watson, y May, 2000). Pero cuál visión es correcta, ¿un optimismo ingenuo o un optimismo realista? La primera generará un peor clima en la institución. La segunda es la correcta. El optimista ve la realidad con claridad, con todos los desafíos y dificultades que tiene, pero tiene la confianza de que algo bueno saldrá (resiliencia), aunque se fracase, se aprenderá para la próxima vez hacerlo mejor (autoeficacia). Es decir, tiene las tres cualidades: es optimista, autoeficaz y resiliente, al mismo tiempo.

Dicho de otra manera:

Los líderes optimistas y resilientes esperan que sus esfuerzos tengan éxito en relación con aquellas cosas sobre las que tienen influencia o control directo, pero no necesariamente que sean lo suficientemente fuertes para superar las fuerzas negativas en sus organizaciones sobre las que tienen poca o ninguna influencia o control; son realistas y optimistas (Leithwood y McCullough, 2020, p.101).

\section{Los optimistas y los "chupaenergías"}

En el estudio de Cross y su equipo (2004) sobre las conexiones entre los empleados, tuvieron la hipótesis de que los empleados con más experiencia y que difundían sus conocimientos serían los considerados como los de máximo rendimiento. No fue así. Descubrieron otro tipo de personas que eran valoradas mucho más para la productividad y eran los energéticos.

Descubrieron que los equipos y organizaciones más prósperos tenían redes plagadas de personas que infundían energía y se relacionaban entre ellas. Desde entonces, Cross y sus colegas han estudiado qué clase de personas transmite energía y por qué triunfan. Los «empleados que infunden energía» no son necesariamente carismáticos o vitales; en apariencia, muchos son sutiles y bastante tímidos. Pero todos generan energía gracias a su optimismo acerca de las posibilidades que se les presentan, involucrando totalmente a la persona que tienen delante en ese momento, valorando las ideas de los demás y ayudando a que la gente sienta que está haciendo progresos (Sutton, 2011, p.74).

Entonces, la visión positiva de las cosas también tiene una relación con la productividad en la gestión de organizaciones. Se sugiere ser líderes que contagian energías antes que chupaenergías o negativos (Sutton, 2007). Entonces es labor del líder convertirse así mismo en alguien que contagia energía positiva y al mismo tiempo, genera estructuras y prácticas escolares en las que se renueve y desarrolle ese optimismo.

\section{Recomendaciones para su desarrollo}

\section{A nivel personal}

Para desarrollar su optimismo a niveles más elevados:

El agradecimiento. Al despertarse cada mañana, piense en tres cosas por las que está agradecido. Solo toma un minuto y le da un giro positivo al día. 
Metas claras. Ya sea que desee un nuevo trabajo o esté lanzando un nuevo proyecto, dar incluso pequeños pasos hacia adelante puede darle una mayor sensación de impulso.

El director César Reina nos recomienda: "Haz volar tus sueños, piensa en aquello que quieras realizar. Separa un pequeño tiempo para hacer un boceto de tus proyectos y cómo llenar de alegría a tus estudiantes, docentes y padres de familia" (Comunicación personal, 16 de noviembre de 2020).

Conéctate con el otro. Siempre busca conectarte con el otro desde el aprecio. Almuerce con amigos que no ha visto últimamente o envíe una nota a un compañero de trabajo con el que está agradecido por algo.

\section{A nivel de equipos}

Se puede desarrollar una cultura del optimismo en la escuela. Algunas recomendaciones de Gielan y Achor (2020):

Modelización. Este es el primer paso para los líderes que quieren crear una mentalidad positiva en medio de las dificultades: modelar esta mentalidad en uno mismo.

Desarrolla un propósito compartido. Una mentalidad positiva en el trabajo es a menudo un ejercicio colectivo porque los comportamientos y actitudes se refuerzan cuando un grupo lo hace en conjunto. Es clave que los líderes ayuden a las personas a sentirse conectadas primero y para lograr luego asumir ser parte de un cambio positivo. Al respecto Nancy Rivera comenta:

Cuando implementamos una estrategia o proyecto o actividad trato en lo posible que surja a partir de las propuestas de todos al menos de un equipo como comisión. Luego se les presenta a todos y se escucha sus sugerencias para reajustarlas. Y si no se modifica algunos aspectos se explica o se dan las razones porque no es posible (Comunicación personal, 3 de diciembre de 2020).

Irma Agurto es directora desde hace siete años del colegio Francisco Izquierdo Ríos en el Callao. Ha sido además especialista de UGEL y formadora de docentes. Sus recomendaciones para desarrollar el optimismo compartido son:

Una de ellas puede ser el tener metas que hayan sido consensuadas y compartidas con todos: Docentes, padre de familia, personal administrativo, de servicio, los exalumnos. De tal manera que, si todos comparten esas metas, nos obliga. Nos obligamos a seguir siendo optimistas. A mantener ese sueño. $Y$ también nos permite animarnos mutuamente, alentarnos. Otra forma es buscar esa gente optimista, identificar a la gente que con una sonrisa te cambia la vida. Juntarte con estas personas, hablar de transformación y recordar siempre y fijar los avances que va teniendo el colegio. De repente en las redes en los grupos de WhatsApp. Ponerlos en grande y compartirlos. Por más pequeño que sea el logro hay que comentarlo (Comunicación personal, 7 de diciembre de 2020).

Ysaac Abarca tiene más de 30 años en el mundo de la gestión educativa. Es docente de educación física y ha sido director de escuelas, especialista pedagógico y director de gestión pedagógica en la Dirección Regional de Educación del Callao. Al respecto comparte su concepción del optimismo en la gestión:

Si parto de que estoy allí para solucionar los problemas y no para generar más problemas, entonces la vida en gestión se hace mucho más digerible [... ]En principio yo me basaba en que todos los problemas tienen solución. Les decía lo siguiente. Yo ya no me apellido Abarca, me apellido solución (Comunicación personal, 16 de diciembre de 2020). 
A continuación, directores de escuelas públicas comparten sus estrategias para desarrollar una cultura del optimismo.

César Reina, director de un colegio del nivel primario aconseja lo siguiente:

Reúnete con frecuencia con tu equipo directivo, docentes y administrativos y comparte una idea, una conversación amena, una lectura, una historia, etc. Alíviales la carga del trabajo cotidiano. Ilumina los espacios de la IE y sorpréndelos con un bonito mensaje, una obra de arte hecho por los estudiantes o una fotografía, que los acompañe por un corto tiempo para que no se deteriore y pierda encanto, luego cámbialo por otro (Comunicación personal, 10 de diciembre de 2020).

Todas las veces que he visitado el colegio de César he comprobado lo que afirma. Aprovecha los espacios al máximo. Al ingreso, cuando se termina de pasar el zaguán en un pequeño espacio del patio se realiza siempre una muestra artística de los niños que va rotando periódicamente.

La directora Nancy Riveros realiza lo siguiente:

En San Marcos se envía todos los lunes al grupo de WhatsApp un saludo positivo agradeciendo a Dios por la oportunidad de iniciar una semana más y los viernes un mensaje deseando a toda la familia sanmarquina un lindo fin de semana. Algo que realizábamos siempre al inicio de las jornadas presenciales era una oración por todo lo que teníamos y habíamos logrado. En la modalidad a distancia también lo estamos haciendo, aunque es un poco impersonal puesto que las no se les ve por las cámaras apagadas (Comunicación personal, 11 de diciembre de 2020).

Como se puede ver estos directores optimistas tratan de insertar esa característica de su personalidad en la institución educativa mediante diversas estrategias según el contexto y características de su grupo humano. De igual manera cada líder debe descubrir e ir probando aquellos rituales o hábitos institucionales que funcionan en su contexto escolar.

\section{Conclusión}

El temperamento o personalidad de los líderes educativos es un factor importante mejorar y realizar cambios en las instituciones educativas y sistemas. De manera específica estas tres características psicológicas deben estar presentes en todo líder educativo a un nivel fuera de lo común: La autoeficacia, la resiliencia y el optimismo. Las tres características se retroalimentan de tal manera que el crecimiento de una ayuda a la otra.

Estas características son difíciles de desarrollar a un nivel elevado es por ello que los reclutadores de directores deben de tomarlas en cuenta para la selección de los mismos. Sin embargo, es posible desarrollarlas si se realiza un esfuerzo consciente y constante. Por lo que las políticas de desarrollo profesional de directivos y líderes educativos deben de incluirlas en sus propuestas de formación. Para identificar el nivel de autoeficacia, resilicencia y optimismo de un postulante se cuentan con pruebas psicológicas validadas así como entrevistas y revisión del portafolio.

Además, se ha podido evidenciar que los directores eficaces tienen estrategias concretas para su desarrollo a nivel personal e institucional. Asimismo, se mostró que cada líder educativo ha encontrado maneras y estrategias diversas para cultivarlas en sí mismos y en la cultura de su institución educativa.

\section{Referencias}

Bandura, A. (1977). Self-efficacy: toward a unifying theory of behavioral change. Psychological Review, 84(2), 191-215. doi: 10.1037/0033-295x.84.2.191 
Bandura, A. (1995). Self-efficacy in changing societies. Cambridge University Press. doi: $10.1017 / C B O 9780511527692$

Bandura, A. (1993). Perceived self-efficacy in cognitive development and functioning. Educational Psychologist, 28(2), 117-148. doi: 10.1207/s15326985ep2802_3

Barrientos, R. (2015). El liderazgo sí importa: aportes para una comprensión de la dirección escolar. Educationis Momentum, 1(1), 83-119. Recuperado de https://revistas.ucsp.edu.pe/index.php/emomentum/article/view/71

Chemers, M. M., Watson, C. B., y May, S. T. (2000). Dispositional Affect and Leadership Effectiveness: A Comparison of Self-Esteem, Optimism, and Efficacy. Personality \& Social Psychology Bulletin, 26(3), 267-277. doi: 10.1177/0146167200265001

Cohen, S., Doyle, W. J., Turner, R. B., Alper, C. M., y Skoner, D. P. (2003). Emotional style and susceptibility to the common cold. Psychosomatic Medicine, 65(4), 652-657. doi: 10.1097/01.psy.0000077508.57784.da

Cross, R. L., y Parker, A. (2004). The Hidden Power of Social Networks: Understanding how Work Really Gets Done in Organizations. Harvard Business Press.

Davis-Blake, A., y Pfeffer, J. (1989). Just a Mirage: The Search for Dispositional Effects in Organizational Research. Academy of Management Review, 14(3), 385-400. doi: 10.5465/amr.1989.4279071

Everson, S. A., Kaplan, G. A., Goldberg, D. E., y Salonen, J. T. (2000). Hypertension incidence is predicted by high levels of hopelessness in Finnish men. Hypertension, 35(2), 561-567. doi: 10.1161/01.hyp.35.2.561

Fullan, M. (2013). Motion Leadership in action. More skinny on becoming change savvy. California, EE. UU.: Corwin Press.

Gielan, M. y Achor, S. (2020). What Leading with Optimism Really Looks Like. EU.: Harvard Business Review. Recuperado de https://hbr.org/2020/06/what-leading-with-optimism-really-looks-like

Harvard Health Publishing. (2008). Optimism and your health. EU.: Harvard Health Publishing. https://www.health.harvard.edu/heart-health/optimism-and-your-health

Leithwood, K., Louis, K. S., Anderson, S. y Wahlstrom, K. (2004). How Leadership Influences Student Learning. Review of research. Recuperado de https://www.inflexion.org/how-leadership-influencesstudent-learning/

Leithwood, K. (2012). Optimism, Resilience and Efficacy [Video]. De: https://thelearningexchange.ca/videos/ken-leithwood-optimism-resilience-and-efficacy/

Leithwood, K., y McCullough, C. (2020). Developing Future School and District Leaders. A Review of Concepts and Evidence. Toronto, Canada: The Learning Partnership.

Scheier, M. F., Matthews, K. A., Owens, J. F., Schulz, R., Bridges, M. W., Magovern, G. J. y Carver, C. S. (1999). Optimism and rehospitalization after coronary artery bypass graft surgery. Archives of Internal Medicine, 159(8), 829-835. doi: 10.1001/archinte.159.8.829

Sutton, R. I. (2007). Weird ideas that work: How to build a creative company. Free Press.

Sutton, R. I. (2011). Buen jefe, mal jefe. Conecta. 